\title{
Characteristics of direct current microhollow cathode discharges combined with dielectric barrier discharges as preionizer
}

\author{
Jun Watanabe, Akihisa Ogino, and Masaaki Nagatsu ${ }^{\text {a) }}$ \\ Graduate School of Science and Technology, Shizuoka University, Hamamatsu, Shizuoka 432-8011, Japan
}

(Received 22 September 2007; accepted 8 November 2007; published online 29 November 2007)

Characteristics of direct current microhollow cathode discharges (MHCDs) combined with dielectric barrier discharge (DBD) electrodes as preionization source were studied experimentally and theoretically. The DBD preionization significantly reduced the sparking voltage (from $3.1-3.2$ to $2.4-2.5 \mathrm{kV}$ ) and improved the spatial uniformity of the multihole MHCDs. Theoretical calculations using the finite element method indicate that the electrons generated by DBDs in the negative phase of the applied voltage could be accelerated into the MHCD electrodes and serve as the seeding electrons for initiating MHCDs. It was experimentally demonstrated that pulselike MHCDs were synchronously triggered by the preionized DBD current pulses. (ㄷ 2007 American Institute of Physics. [DOI: 10.1063/1.2819533]

Atmospheric-pressure discharges have been widely investigated in recent years in order to develop lowtemperature materials processing techniques for a variety of industrial applications, such as surface treatment, thin film deposition, detoxification of gaseous pollution, sterilization, and gas lasers. One of the most important advantages of atmospheric-pressure plasma technology is that it does not require a costly pump system. So far, several methods have been developed to generate atmospheric-pressure plasmas, such as dielectric barrier discharge (DBD) ${ }^{1-4}$ microhollow cathode discharge (MHCD), ${ }^{5-9}$ and plasma jet. ${ }^{10,11}$ As mentioned above, atmospheric-pressure plasmas have been studied with an eye toward developing many different industrial applications. The plasma generation method to be used is selected among the various atmospheric-pressure plasma methods available on the basis of the specific application. In the present study, we investigated multihole type MHCDs to develop atmospheric plasma processing techniques for air and liquid disinfection and sterilization, where the MHCDs can be employed as spatial filters to process the gas or liquid flowing through the holes.

In the multihole electrode, it is generally difficult to sustain MHCDs uniformly over all the holes. Furthermore, a high interelectrode voltage is required to ignite the atmospheric pressure plasma depending on the interelectrode distance. Hence, we propose to use the DBD electrodes as preionizer for the multihole MHCD system. The effects of DBD preionization on lowering the ignition voltage are first studied using a single-hole MHCD, and then the discharge uniformity is improved using multihole MHCDs. To gain a physical understanding of the combined MHCD-DBD electrode system, we also performed a theoretical calculation of equipotential distributions in the present configuration using the finite element method. Experimental results of the effect of DBD on the ignition of MHCDs were discussed on the basis of the theoretical calculations.

Figure 1 schematizes the combined MHCD-DBD electrode system, connected to a dc or ac power source. The MHCD electrodes consisted of two molybdenum sheets, $0.1 \mathrm{~mm}$ in thickness, and a $0.5-\mathrm{mm}$-thick mica sheet as a

a) Tel.: +81-53-478-1081. Electronic mail: tmnagat@ipc.shizuoka.ac.jp. spacer. There were seven holes, each is $0.3 \mathrm{~mm}$ in diameter, formed by using a mechanical drill. The MHCD was generated by applying a dc voltage between the electrodes. A typical current limiting resistance of $867 \mathrm{k} \Omega$ was used to prevent the transition from glow to arc.

A high voltage dc power supply with a maximum current of $2 \mathrm{~A}$ and a maximum voltage of $15 \mathrm{kV}$ was used in the present experiment. The output voltage of the dc power supply was varied manually. As shown in Fig. 1, the DBD electrode was set near the cathode electrode of the MHCD. The DBD was generated by applying $1-7 \mathrm{kHz}$ sinusoidal waves using an ac amplifier with a maximum output voltage of $10 \mathrm{kV}$. The voltage and current between the MHCD electrodes measured by a high voltage divider and a current monitoring low resistance were simultaneously recorded by a digital oscilloscope.

Figure 2 shows the discharge characteristics of a singlehole MHCD with and without DBD preionization under a ballast resistance of $867 \mathrm{k} \Omega$. Manually increasing the $\mathrm{dc}$ voltage to $2.0 \mathrm{kV}$ without DBD preionization led to unstable oscillatory discharges. A similar oscillating behavior in $I-V$ characteristics has been observed by others. ${ }^{12,13}$ It is thought that self-pulsing phenomena occur only if the load line crosses the discharge characteristics in the region of negative differential resistivity. ${ }^{12}$ Our experiment confirmed that the oscillating behavior was suppressed when the ballast resistance was lower than about $200 \mathrm{k} \Omega$. When the applied voltage was increased to about $2.9-3.0 \mathrm{kV}$, the interelectrode voltage in the unstable $V-I$ regime dropped to about $500 \mathrm{~V}$ in

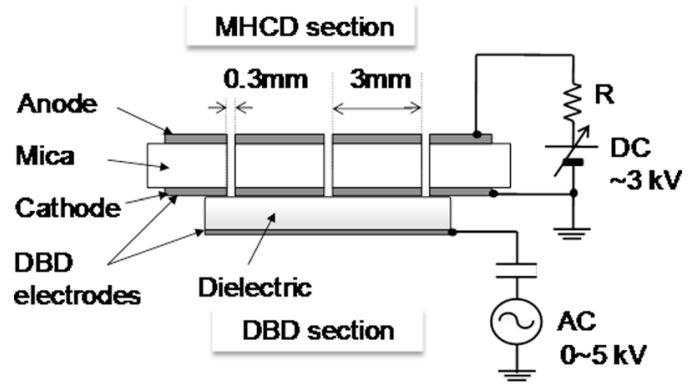

FIG. 1. Schematic drawing of the combined MHCD-DBD electrode system. 
(a)

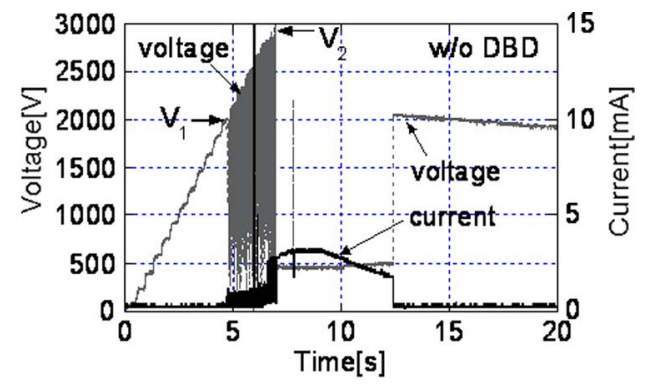

(b)

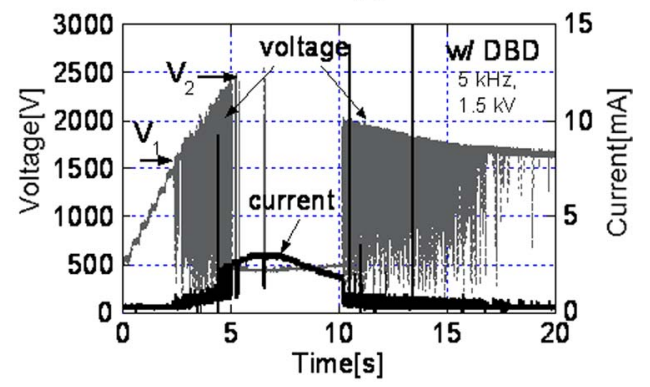

FIG. 2. (Color online) Comparison of the ignition of single-hole MHCD (a) without and (b) with DBD preionization.

the normal glow regime and a stable glow discharge was obtained. As shown in Fig. 2, the voltage at the starting point of the unstable discharge was denoted by $V_{1}$, and that at the transition from oscillatory discharge to stable glow discharge by $V_{2}$.

By contrast, in the case of "with DBD preionization," an unstable discharge started at $V_{1} \sim 1.5 \mathrm{kV}$ and a transition from abnormal to normal glow discharge occurred at $V_{2}$ $\sim 2.5 \mathrm{kV}$ under similar experimental conditions as in the case of "without DBD preionization." Both transitions occurred at roughly $500 \mathrm{~V}$ below the voltage observed in the case of without DBD preionization.

To confirm the effect of DBDs as preionizer, we changed the ac frequency, keeping the applied amplitude voltage constant at $\pm 1.5 \mathrm{kV}$. Then, the amplitudes of the displacement and spiky currents of the DBDs increased with the frequency of the applied ac signals. This is because the DBD displacement current is proportional to the frequency at a fixed amplitude voltage. Figure 3 shows the dependence of the DBD frequency on the voltages $V_{1}$ and $V_{2}$. At a fixed ac amplitude voltage of $\pm 1.5 \mathrm{kV}$, the frequency was varied from 1 to $7 \mathrm{kHz}$. In Fig. 3, the values of $V_{1}$ and $V_{2}$ for the case of without DBD preionization were plotted at zero DBD fre-

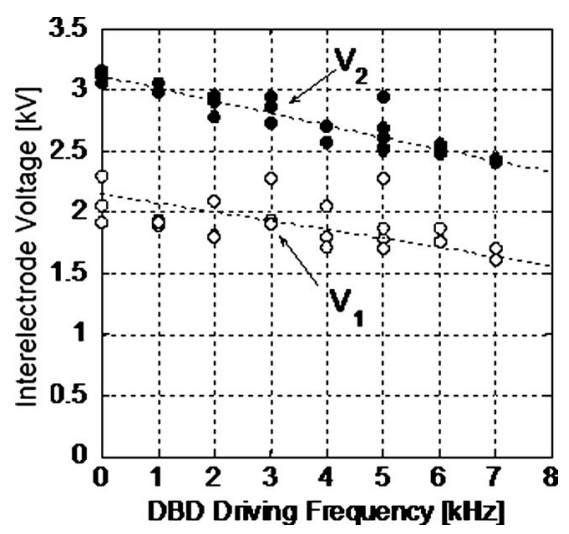

FIG. 3. The relation between the ignition voltages $V_{1}$ and $V_{2}$ and DBD frequency at a fixed voltage of $\pm 1.5 \mathrm{kV}$.

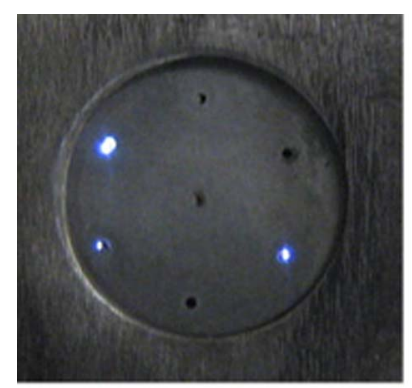

(a) wo DBD

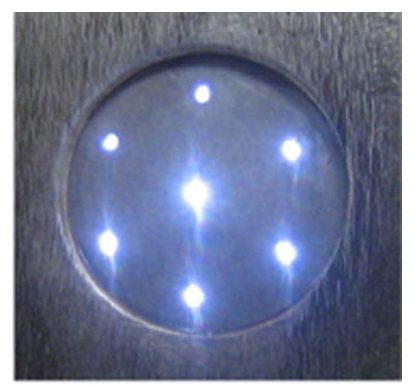

(b) $\quad$ w/DBD(5kHz, 1,5kV)

FIG. 4. (Color online) Photographs of microhollow discharge (a) without and (b) with DBD preionization.

quency for the sake of convenience. Figure 3 shows that both $V_{1}$ and $V_{2}$ decreased with DBD frequency. At an ac frequency of $7 \mathrm{kHz}$, for example, the ignition voltage $V_{2}$ clearly decreased from $3.1-3.2 \mathrm{kV}$ in the case of without DBD to $2.4-2.5 \mathrm{kV}$. The present results clearly show that the additional DBD helps us to ignite MHCD at much lower voltages. Similar results were obtained when the DBD driving voltage was increased at a fixed frequency. It was observed that $V_{2}$ suddenly dropped from 3.2 to $2.5-2.7 \mathrm{kV}$ as soon as the DBD started at an ac amplitude voltage exceeding roughly $\pm 1.0 \mathrm{kV}$ at $7 \mathrm{kHz}$. These results suggest that the electrons generated by DBD serve as the primary electrons for the ignition of MHCD.

In order to demonstrate the effect of DBD preionization on multihole MHCDs, we used a seven-hole MHCD structure (see Fig. 1). Figure 4 shows photographs of multihole MHCDs with and without DBD preionization taken by a digital camera with a shutter speed of $1 / 8 \mathrm{~s}$. Without DBD, only two or three microhollow discharges were observed, as shown in Fig. 4(a). By contrast, with DBD as preionization, we observed uniform discharges in all the holes, as shown in Fig. 4(b). These uniform multihole discharges were obtained during the unstable discharge period. After transition to the glow discharge regime, the MHCD was sustained at only one hole. This may be because the present geometry of multihole MHCD electrodes is not the same for all the holes.

To study in detail the effect of DBD on the electric field distribution in the MHCD section, we carried out a field analysis using the finite element method. Here, we assumed that a dc voltage of $1.5 \mathrm{kV}$ was applied between the MHCD electrodes, where the cathode was grounded. We changed the voltage of the DBD electrode with respect to the grounded electrode. Figure 5 shows the calculation results, where the potentials of the DBD powered electrode were +0.75 and $-0.75 \mathrm{kV}$. When the voltage of the lower DBD electrode was $+0.75 \mathrm{kV}$, radial electric fields were formed inside the cathode hole, as shown in Fig. 5. In such an equipotential distribution, positive ions generated by DBD are accelerated toward the inner surface of the cathode hole. It is thought that the radial electric field in the cathode hole is essential in sustaining the hollow cathode discharge. However, the charged particles generated by DBDs, especially electrons, did not contribute to MHCD ignition because sufficient electrons were not introduced into the interelectrode region of MHCD. On the other hand, when the voltage of the powered DBD electrode was $-0.75 \mathrm{kV}$, equipotential lines near the cathode of the MHCD section are parallel to the electrode surface and form the axial electric field, which accelerates AIP license or copyright; see http://apl.aip.org/apl/copyright.jsp 


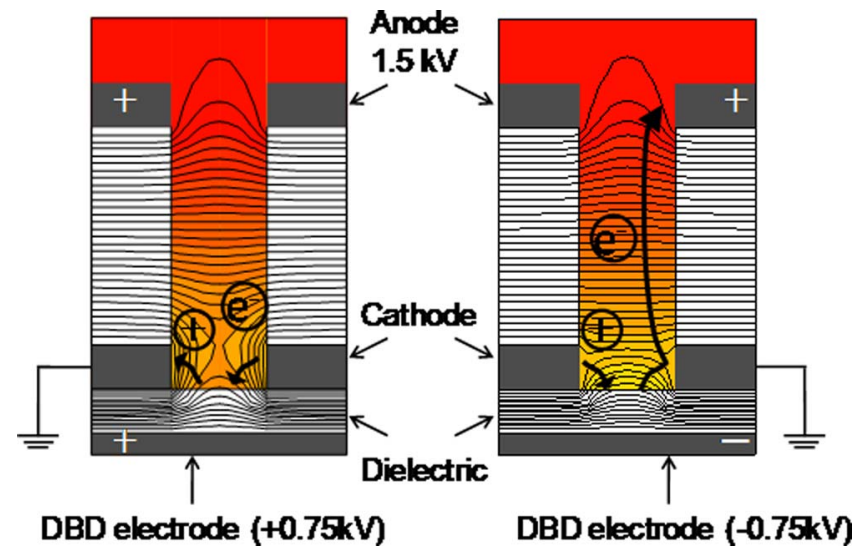

FIG. 5. (Color online) Calculation results of the finite element method for explaining the role of DBD preionization in the present MHCD system.

electrons toward the anode of the MHCD section. In the present combined MHCD-DBD system, electrons generated by DBD near the cathode of MHCD will be accelerated toward the anode of the MHCD section. If enough electrons are introduced into the interelectrode region, there will be a discharge between the MHCD electrodes.

To examine the effect of DBD preionization on the ignition voltage of MHCD experimentally, we briefly discuss the DBD-driven oscillatory discharge phenomena observed before the transition from the unstable discharge to the stable glow discharge. These synchronously oscillatory discharges are quite different from the self-pulsing discharges observed in Fig. 2(a). Figure 6 shows the typical temporal behavior of the applied sinusoidal DBD signal and corresponding MHCD voltage waveform. It is interesting to note that the interelectrode voltage between MHCD electrodes suddenly

(a)

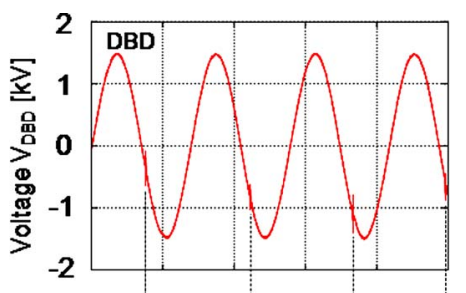

(b)

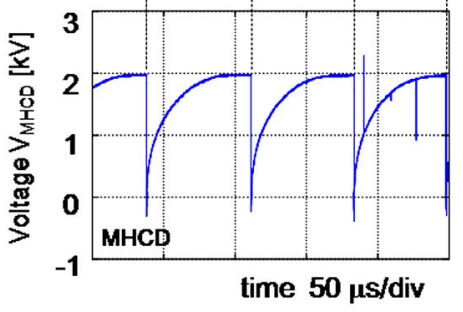

FIG. 6. (Color online) Temporal behavior of (a) the applied DBD voltage and (b) corresponding MHCD voltage waveform. dropped from about $2.0 \mathrm{kV}$ to almost zero at the DBD current spikes generated in the negative polarity phase having a negative derivative, $d V / d t$. When we decreased the frequency of the ac source down to $500 \mathrm{~Hz}$, keeping the driver voltage at $1.5 \mathrm{kV}$, the DBD stopped. Through this lowfrequency operation, we confirmed that the discharge of the MHCD section no longer occurred, even though the DBD amplitude voltage was the same. This result agrees with the results of the finite element method and clearly indicates that the initial electrons generated by DBD play an essential role in triggering the MHCDs.

In conclusion, we presented the discharge characteristics of MHCDs with a multihole structure. We employed DBD as preionization source for the multihole MHCD system. The effects of DBD preionization on lowering the ignition voltage and improving the discharge uniformity were investigated using single-hole microhollow cathode electrodes. To gain a physical understanding of the combined MHCD-DBD electrode system, we performed a theoretical calculation of equipotential distributions in the present configuration using the finite element method. We presented preliminary experimental results on the multihole MHCD and theoretical calculation results of field analysis using the finite element method in the present configuration. With DBD preionization, we confirmed that MHCDs were generated at about $500 \mathrm{~V}$ below the voltages observed in the case of without DBD.

This work was partly supported by a Grant-in-Aid for Scientific Research from Japan Society for the Promotion of Science.

${ }^{1}$ S. Kanazawa, M. Kogoma, T. Moriwaki, and S. Okazaki, J. Phys. D 21, 863 (1988)

${ }^{2}$ S. Okazaki, M. Kogoma, M. Uehara, and Y. Kimura, J. Phys. D 26, 889 (1993).

${ }^{3}$ F. Massines, A. Rabehi, P. Decomps, R. B. Gadri, P. Segur, and C. Mayoux, J. Appl. Phys. 83, 2950 (1998).

${ }^{4}$ B. Eliasson and U. Kogelschatz, Appl. Phys. B: Photophys. Laser Chem. 46, 299 (1988).

${ }^{5}$ K. H. Schoenbach, A. El-Habachi, W. Shi, and M. Ciocca, Plasma Sources Sci. Technol. 6, 468 (1997).

${ }^{6}$ R. H. Stark and K. H. Schoenbach, J. Appl. Phys. 15, 2075 (1999).

${ }^{7}$ R. H. Stark and K. H. Schoenbach, Appl. Phys. Lett. 74, 3770 (1999).

${ }^{8}$ J. Chen, S.-J. Park, Z. Fan, and J. G. Eden, J. Microelectromech. Syst. 11, 536 (2002).

${ }^{9}$ K. H. Schoenbach, M. Moselhy, A. Shi, and R. Bentley, J. Vac. Sci. Technol. A 21, 1260 (2003).

${ }^{10}$ A. Schutze, J. Y. Jeong, S. E. Babayan, J. Park, G. S. Selwyn, and R. F. Hicks, IEEE Trans. Plasma Sci. 26, 1685 (1998).

${ }^{11}$ J. Park, I. Henins, H. W. Herrmann, G. S. Selwyn, J. Y. Jeong, R. F. Hicks, D. Shim, and C. S. Chang, Appl. Phys. Lett. 76, 288 (2000).

${ }^{12}$ D. D. Hsu and D. B. Graves, J. Phys. D 36, 2898 (2003).

${ }^{13}$ X. Aubert, G. Bauville, J. Guillon, B. Lacour, V. Puech, and A. Roussseau, Plasma Sources Sci. Technol. 16, 23 (2007). 